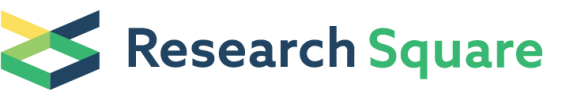

Preprints are preliminary reports that have not undergone peer review. They should not be considered conclusive, used to inform clinical practice, or referenced by the media as validated information.

\section{Real-World Efficacy and Safety of Pangenotypic Direct-Acting Antivirals Against Hepatitis C Virus Infection in Taiwan}

\section{Kao-Chi Chang}

Chang Gung Memorial Hospital Chiayi Branch: Chiayi Chang Gung Memorial Hospital https://orcid.org/0000-0002-0185-2286

\section{Shui-Yi Tung}

Chang Gung Memorial Hospital Chiayi Branch: Chiayi Chang Gung Memorial Hospital

\section{Kuo-Liang Wei}

Chang Gung Memorial Hospital Chiayi Branch: Chiayi Chang Gung Memorial Hospital

\section{Chen-Heng Shen}

Chang Gung Memorial Hospital Chiayi Branch: Chiayi Chang Gung Memorial Hospital

\section{Yung-Yu Hsieh}

Chang Gung Memorial Hospital Chiayi Branch: Chiayi Chang Gung Memorial Hospital

\section{Wei-Ming Chen}

Chang Gung Memorial Hospital Chiayi Branch: Chiayi Chang Gung Memorial Hospital

\section{Yi-Hsing Chen}

Chang Gung Memorial Hospital Chiayi Branch: Chiayi Chang Gung Memorial Hospital

\section{Chun-Hsien Chen}

Chang Gung Memorial Hospital Chiayi Branch: Chiayi Chang Gung Memorial Hospital Chi-Wei Yen

Chang Gung Memorial Hospital Chiayi Branch: Chiayi Chang Gung Memorial Hospital Huang-Wei Xu

Chang Gung Memorial Hospital Chiayi Branch: Chiayi Chang Gung Memorial Hospital

\section{Wei-Lin Tung}

Chang Gung Memorial Hospital Chiayi Branch: Chiayi Chang Gung Memorial Hospital

\section{Chao-Hung Hung}

Chang Gung Memorial Hospital Chiayi Branch: Chiayi Chang Gung Memorial Hospital

\section{Sheng-Nan Lu}

Chang Gung Memorial Hospital Chiayi Branch: Chiayi Chang Gung Memorial Hospital

Te-Sheng Chang ( $\square$ cgmh3621@cgmh.org.tw)

Chang Gung Memorial Hospital Chiayi Branch: Chiayi Chang Gung Memorial Hospital 
Keywords: glecaprevir, pibrentasvir, sofosbuvir, velpatasvir, sustained virologic response, ribonucleic acid virus, cirrhosis, hepatocellular carcinoma, genotype variants, ribavirin

Posted Date: February 17th, 2021

DOI: https://doi.org/10.21203/rs.3.rs-202089/v1

License: (1) This work is licensed under a Creative Commons Attribution 4.0 International License. Read Full License 


\section{Abstract}

Background/Purpose of Study

Clinical trials have demonstrated excellent efficacy and safety of pangenotypic direct-acting antivirals (DAAs) for the treatment of hepatitis $\mathrm{C}$ virus (HCV). However, there are limited large-scale real-world data on these pangenotypic DAAs. This study examined the results of two pangenotypic regimens, glecaprevir/pibrentasvir (GLE/PIB) and sofosbuvir/velpatasvir (SOF/VEL), in a real-world setting in Taiwan.

Methods

All HCV patients treated with GLE/PIB or SOF/VEL from August 2018 to April 2020 at the Chiayi Chang Gung Memorial Hospital were included. The primary end point was sustained virologic response 12 weeks after treatment cessation (SVR12). Adverse events (AEs) were also reported.

Results

A total of $1356 \mathrm{HCV}$ patients received pangenotypic DAA treatment during the study period: 742 patients received GLE/PIB and 614 received SOF/VEL. The rates of SVR12 for GLE/PIB and SOF/VEL were 710/718 (98.9\%) and 581/584 (99.5\%), respectively, by per-protocol analysis, and 710/742 (95.7\%) and $581 / 614$ (94.6\%), respectively, by evaluable population analysis. Eleven patients (GLE/PIB: 8, SOF/VEL: 3) did not achieve SVR12. The most common AEs for GLE/PIB and SOF/VEL were pruritus ( $17.4 \%$ vs. $2.9 \%$ ), abdominal discomfort (5.8\% vs. $4.4 \%$ ), dizziness ( $4.2 \%$ vs. $2 \%$ ), and malaise ( $3.1 \%$ vs. $2.9 \%)$. Laboratory abnormalities were uncommon, with $<1 \%$ of patients exhibiting elevated total bilirubin or aminotransferase levels with both regimens. There were five drug discontinuations owing to AEs (bilirubin elevation: 3, dermatological issues: 2).

Conclusion

In real-world practice, the pangenotypic DAAs GLE/PIB and SOF/VEL are effective and well tolerated, achieving high SVR12 rates for patients with all HCV genotypes.

\section{Introduction}

Hepatitis $\mathrm{C}$ virus ( $\mathrm{HCV}$ ) infection is a major global public health problem and is associated with serious complications, including cirrhosis, hepatocellular carcinoma (HCC), and mortality. According to the global hepatitis report published by the World Health Organization, HCV infected 71 million people worldwide and caused $~ 400000$ deaths in 2015[1]. The goal of HCV infection treatment is to achieve a sustained virological response (SVR). An SVR is defined as the absence of detectable levels of HCV ribonucleic acid (RNA) in the blood at least 12 weeks after completing treatment. An SVR after treatment with direct-acting antivirals (DAAs), or interferon plus ribavirin, significantly decreases the complications of liver disease, extrahepatic manifestations, risk of HCC, and mortality[2-6]. 
$\mathrm{HCV}$ is a minute enveloped single-stranded virus of $9600-\mathrm{kb}$ RNA belonging to the Flaviviridae family. There are seven known HCV genotypes with genome differences of more than $30 \%$ at the nucleotide level. Genotypes 1-6 are the most studied variants[7]. Based on the rapid progress in the treatment of HCV infection in recent years, interferon-free DAAs have proven to be effective and safe and have become the standard of care for HCV infection[8]. The choice of a suitable DAA regimen is complex initially, and the $\mathrm{HCV}$ genotype is one of the major factors determining an appropriate genotype-specific DAA. With the advent of pangenotypic DAA regimens, hepatitis $C$ treatment has been simplified, and HCV genotyping is no longer an absolute requirement before initiating treatment[9].

Glecaprevir/pibrentasvir (GLE/PIB) (100/40 mg, Maviret, Fournier Laboratories Ireland Limited Anngrove, Carrigtwohill, Cork, Ireland) is a fixed-dose combination (FDC) of two pangenotypic DAAs: GLE, a nonstructural viral protein 3/4A (NS3/4A) protease inhibitor, and PIB, a novel next-generation nonstructural viral protein $5 \mathrm{~A}$ (NS5A) inhibitor with potent pangenotypic activity with or without compensated cirrhosis in patients aged $\geq 12$ years[10-13]. Depending on the patient's genotype, whether cirrhosis is present, and prior treatment experience, the recommended dose is three tablets (GLE 300 mg/PIB $120 \mathrm{mg}$ ) once daily for eight or 12 weeks[9]. Sofosbuvir/velpatasvir (SOF/VEL) (400/100 mg, Epclusa, Gilead Sciences Inc., Foster City, CA) is also an FDC of two pangenotypic DAAs: SOF, a livertargeted pyrimidine nucleotide analog that functions as a chain terminator and inhibits nonstructural viral protein 5B (NS5B) RNA-dependent RNA polymerase with pangenotypic potency, and VEL, a potent pangenotypic NS5A inhibitor. SOF/VEL is approved for chronic HCV genotypes 1 through 6, without ribavirin, in patients aged $\geq 6$ years with or without compensated cirrhosis[14-18]. It is administered using a weight-based dosing regimen of ribavirin in patients with decompensated cirrhosis. The recommended dose is one tablet (SOF $400 \mathrm{mg} / \mathrm{VEL} 100 \mathrm{mg}$ ) once daily for 12 weeks[9].

Previous clinical trials have demonstrated excellent efficacy and safety of both GLE/PIB and SOF/VEL in their respective patient cohorts. However, large-scale, real-world data involving these two pangenotypic DAAs are still limited. We evaluated the real-world efficacy and safety of GLE/PIB and SOF/VEL in a large cohort of patients with HCV infection of various genotypes in Taiwan.

\section{Methods}

\section{Patients}

In Taiwan, the National Health Insurance (NHI) has funded DAA treatment of patients with HCV infections since 2017. Between 2017 and 2018, this nationwide government-funded program enrolled only chronic HCV-infected patients with a hepatic fibrosis stage $\geq$ F3. However, in 2019, Taiwan's NHI extended its coverage and approved treatment of all HCV viremic patients regardless of the severity and duration of liver disease. GLE/PIB was approved by Taiwan's Food and Drug Administration (TFDA) on January 19, 2018 and reimbursed by Taiwan's NHI on August 1,2018. SOF/VEL was approved by the TFDA on December 17, 2018 and reimbursed by Taiwan's NHI on June 1, 2019. 
Between August 2018 and April 2020, all patients aged $\geq 18$ years with quantifiable serum HCV RNA (Cobas AmpliPrep/Cobas TaqMan HCV quantitative test v2.0, Roche Molecular Systems Inc., Pleasanton, CA, USA, lower limit of detection [LLOD]: $15 \mathrm{IU} / \mathrm{mL}$ across all HCV genotypes) who received GLE/PIB for 8 or 12 weeks or SOF/VEL for 12 weeks, with or without ribavirin, at Chiayi Chang Gung Memorial Hospital $(\mathrm{CGMH})$ were included in the study.

This study was approved by the Institutional Review Board of Chang Gung Medical Foundation on April 15, 2019 (IRB No. : 201900542B0) and was conducted in accordance with the principles of the Declaration of Helsinki and the International Conference on Harmonization for Good Clinical Practice. All patients gave their informed consent prior to their inclusion in the study.

\section{Study design}

The following data were collected for all patients: basic demographics, prior treatment experience, prior history of HCC, presence of diabetes mellitus, hepatitis B virus (HBV) surface antigen, HCV RNA quantification, HCV genotype (Cobas HCV GT kit based on the Cobas 4800 system from Roche Diagnostics, Roche Molecular Systems Inc., Pleasanton, CA, USA), fibrosis index based on four factors (FIB-4), hemogram, serum albumin, total bilirubin, aspartate aminotransferase (AST), alanine aminotransferase (ALT), creatinine, estimated glomerular filtration rate (eGFR), and alpha-fetoprotein. Clinical evidence of hepatic fibrosis or cirrhosis was determined based on the FIB-4, acoustic radiation force impulse (ARFI) (Siemens Acuson S2000, Virtual Touch ${ }^{\text {TM }}$ tissue quantification; Siemens), or the presence of portal hypertension based on image examinations.

The treatment regimen and duration were determined according to the current international guidelines for $\mathrm{HCV}$ infection and at the discretion of the treating physician. Patients with HCV genotypes 1-6 without liver decompensation received three oral GLE/PIB tablets with food once daily for eight or 12 weeks. When SOF/VEL was prescribed for HCV genotype 1-6 patients, the treatment duration was universally 12 weeks, and weight-based ribavirin (Robatrol, $200 \mathrm{mg}$ capsule, Genovate Biotechnology Co. Ltd.) was added for patients with decompensated cirrhosis. All patients received follow-up for a minimum of 12 weeks after completing treatment.

\section{Assessment of efficacy}

The primary efficacy end point was the SVR rate at 12 weeks after completing treatment (SVR12). The SVR12 rate was defined as the proportion of patients who had serum HCV RNA levels less than the LLOD at off-treatment week 12. The SVR12 rates in both the evaluable population (EP) (patients who received at least one dose of GLE/PIB or SOF/VEL) and per-protocol population (PP) (patients who received at least one dose of GLE/PIB or SOF/VEL, and whose HCV RNA data at post-therapy week 12 was available) were reported. The secondary efficacy end point was the rate of HCV RNA levels less than the LLOD at the end of treatment (EOT). Non-response was defined as detectable HCV RNA at the EOT. Relapse was defined as undetectable HCV RNA levels at the EOT, but detectable HCV RNA at off-treatment week 12.

\section{Assessment of safety}


The payment regulations of Taiwan's NHI stipulate that patients receiving DAA therapy must return to the hospital at least every four weeks during treatment and three months after completing treatment. Safety was evaluated using anamnesis, physical examination, and laboratory tests, and recorded in the CGMH electronic medical records database by physicians during every visit. Contraindicated drug-drug interactions (DDIs) between GLE/PIB or SOF/VEL and concomitant medications were assessed before and during the therapeutic course. In patients who experienced serious AEs or discontinued treatment, the causal relationship between the events and DAAs and the reasons for discontinuation were reviewed from the medical records. The following were considered serious treatment-related AEs: death; hepatic decompensation (variceal hemorrhage, ascites, hepatic encephalopathy, or prolonged prothrombin time > $3 \mathrm{~s}$ ); grade 3 hyperbilirubinemia (total bilirubin levels more than 3 times above the upper limit of the normal (ULN) range); and HBV reactivation (presence of an HBV DNA level $\geq$ LLOD in patients with undetectable baseline HBV DNA or an increase in the HBV DNA level to $\geq 1 \log _{10} \mathrm{IU} / \mathrm{mL}$ in patients with detectable baseline HBV DNA).

\section{Statistical analysis}

Continuous data are expressed as means \pm standard deviations or medians and ranges. Dichotomous data are expressed as numbers (percentages). Qualitative and quantitative differences between groups were analyzed using the chi-square test for dichotomous variables and the Student's t-test or MannWhitney U test for continuous variables, as appropriate. IBM SPSS Statistics for Windows, version 22.0 (IBM Corp., Armonk, N.Y., USA) was used for statistical analysis. Statistical significance was set as a twotailed $P$ value $<0.05$.

\section{Results}

\section{Patient characteristics}

Between August 16, 2018 and April 22, 2020, a total of 1356 patients with HCV viremia received pangenotypic DAA treatment at the CGMH. A total of 742 patients received GLE/PIB and 614 received SOF/VEL. Baseline patient characteristics for both the GLE/PIB and SOF/VEL groups were similar (Table 1). The greatest differences between the two groups were the use of ribavirin and treatment durations. The patients in the GLE/PIB group did not require ribavirin and most patients without cirrhosis underwent an 8-week treatment course, whereas all patients in the SOF/VEL group received 12 weeks of therapy, and ribavirin was required for patients with decompensated liver cirrhosis. The number of treatment-experienced patients in the GLE/PIB group $(n=96,12.9 \%)$ was higher than that in the SOF/VEL group $(n=28,4.5 \%)$. This is because GLE/PIB was approved by the TFDA and reimbursed by Taiwan's $\mathrm{NHI}$ earlier than SOF/VEL. Thus, most patients who experienced failure of antiviral therapy had received GLE/PIB as soon as it started being reimbursed. Another significant difference was the higher baseline creatinine levels in the GLE/PIB group. Most patients with renal function impairment received GLE/PIB before the formal approval of SOF/VEL for the treatment of patients with chronic kidney disease. 
Table 1

Baseline characteristics of patients

\begin{tabular}{|c|c|c|c|}
\hline & GLE/PIB & SOF/VEL & $p$ value \\
\hline Number & 742 & 614 & \\
\hline Age, year, mean $\pm S D$ & $62.12 \pm 12.95$ & $63.31 \pm 14.24$ & 0.111 \\
\hline Sex, Male/Female & $375 / 367$ & $290 / 324$ & 0.225 \\
\hline Treatment experience & & & $<0.001$ \\
\hline Naïve/Experienced & $646 / 96$ & $586 / 28$ & \\
\hline HBV coinfection & & & 0.181 \\
\hline Absent/Present & $676 / 66$ & $546 / 68$ & \\
\hline Prior HCC history & & & 0.348 \\
\hline Absent/Present & $714 / 28$ & $583 / 31$ & \\
\hline Treatment duration, week & & & $<0.001$ \\
\hline $8 / 12$ & $622 / 120$ & $0 / 614$ & \\
\hline Ribavirin usage & & & $<0.001$ \\
\hline No/Yes & $742 / 0$ & $588 / 26$ & \\
\hline Diabetes mellitus & & & $<0.001$ \\
\hline No/Yes & $523 / 219$ & $485 / 129$ & \\
\hline HCV RNA, IU/mL & & & 0.471 \\
\hline$<800,000 / \geq 800,000$ & $235 / 507$ & $203 / 411$ & 0.586 \\
\hline \multirow[t]{2}{*}{ HCV RNA, IU/mL, median (range) } & $2,282,480$ & $1,777,346$ & 0.047 \\
\hline & $\begin{array}{l}(18- \\
40,230,995)\end{array}$ & $\begin{array}{l}(51- \\
34,466,974)\end{array}$ & \\
\hline HCV genotype & & & Total \\
\hline $1 \mathrm{a}$ & 20 & 16 & $36(2.65 \%)$ \\
\hline $1 b$ & 138 & 248 & $386(28.47 \%)$ \\
\hline 2 & 492 & 296 & $788(58.11 \%)$ \\
\hline
\end{tabular}

eGFR: estimated glomerular filtration rate; FIB-4: fibrosis index based on four factors; SD: standard deviation; GLE/PIB: glecaprevir/pibrentasvir; SOF/VEL: sofosbuvir/velpatasvir; HCV: hepatitis C virus; HBV: hepatitis B virus; HCC: hepatocellular carcinoma; RNA: ribonucleic acid; AST: aspartate aminotransferase; ALT: alanine aminotransferase 


\begin{tabular}{|c|c|c|c|}
\hline & GLE/PIB & SOF/VEL & $p$ value \\
\hline 3 & 12 & 4 & $16(1.18 \%)$ \\
\hline 6 & 17 & 12 & $29(2.14 \%)$ \\
\hline Mixed & 63 & 38 & $101(7.45 \%)$ \\
\hline FIB- $4,>3.25 / \leq 3.25$ & $160 / 582$ & $143 / 471$ & 0.447 \\
\hline Hemoglobin, g/dL, mean $\pm S D$ & $13.67 \pm 1.88$ & $13.52 \pm 1.89$ & 0.138 \\
\hline $\begin{array}{l}\text { White blood cell count, } 10^{9} \text { cells } / \mathrm{L} \text {, } \\
\text { mean } \pm S D\end{array}$ & $6.34 \pm 1.93$ & $6.52 \pm 4.03$ & 0.283 \\
\hline Platelet count, $10^{9}$ cells $/ \mathrm{L}$, mean $\pm S D$ & $197.20 \pm 63.17$ & $198.93 \pm 77.40$ & 0.658 \\
\hline Albumin, $\mathrm{g} / \mathrm{dL}$, mean $\pm S D$ & $4.28 \pm 0.38$ & $4.18 \pm 0.50$ & $<0.001$ \\
\hline Total bilirubin, mg/dL, mean $\pm S D$ & $0.83 \pm 0.35$ & $0.98 \pm 0.83$ & $<0.001$ \\
\hline $\mathrm{AST}, \mathrm{U} / \mathrm{L}$, mean $\pm \mathrm{SD}$ & $48.85 \pm 38.94$ & $51.37 \pm 45.26$ & 0.270 \\
\hline $\mathrm{ALT}, \mathrm{U} / \mathrm{L}$, mean $\pm \mathrm{SD}$ & $60.72 \pm 58.22$ & $61.46 \pm 67.80$ & 0.829 \\
\hline Creatinine, $\mathrm{mg} / \mathrm{dL}$, mean $\pm S D$ & $1.38 \pm 1.92$ & $0.96 \pm 0.78$ & $<0.001$ \\
\hline eGFR, $\mathrm{mL} / \mathrm{min} / 1.73 \mathrm{~m}^{2}$, mean $\pm S D$ & $80.54 \pm 30.71$ & $84.94 \pm 27.39$ & 0.005 \\
\hline Alpha-fetoprotein, ng/mL, median (range) & $3.1(0.5-3455.3)$ & 3.3(0.7-16509.5) & 0.039 \\
\hline \multicolumn{4}{|c|}{$\begin{array}{l}\text { eGFR: estimated glomerular filtration rate; FIB-4: fibrosis index based on four factors; SD: standard } \\
\text { deviation; GLE/PIB: glecaprevir/pibrentasvir; SOF/VEL: sofosbuvir/velpatasvir; HCV: hepatitis C virus; } \\
\text { HBV: hepatitis B viruS; HCC: hepatocellular carcinoma; RNA: ribonucleic acid; AST: aspartate } \\
\text { aminotransferase; ALT: alanine aminotransferase }\end{array}$} \\
\hline
\end{tabular}

\section{Efficacy}

Of the 1356 patients who received pangenotypic DAA therapy, 1318 completed the treatment course and had available EOT data. Thirty-eight patients did not complete the therapeutic protocol. The flowchart of patient enrollment and withdrawal is presented in Fig. 1. Ten of these 38 patients achieved SVR12. A total of 29 patients were withdrawn from the pangenotypic DAA treatment: 16 in the GLE/PIB group and 13 in the SOF/VEL group. The reasons for patient withdrawal are shown in Table 2. At the EOT, 724 and 593 patients in the GLE/PIB and SOF/VEL groups, respectively, had undetectable serum HCV RNA levels. By EP analysis, the SVR12 rates were 710/742 (95.7\%) and 581/614 (94.6\%) in the GLE/PIB and SOF/VEL groups, respectively. By PP analysis, the SVR12 rates were 710/718 (98.9\%) and 581/584 (99.5\%) in the GLE/PIB and SOF/VEL groups, respectively (Table 3). Eleven patients did not attain SVR12: eight received GLE/PIB (relapses: 7, non-responder: 1) and three received SOF/VEL (non-responders: 3). 
Table 2

Reasons for DAA withdrawal

\begin{tabular}{|lll|}
\hline Reasons for withdrawal & GLE/PIB $(\mathbf{N}=16)$ & SOF/VEL $(\mathbf{N}=13)$ \\
\hline Lost to follow-up & 7 & 10 \\
\hline Forget to come back & 1 & \\
\hline Refuse to check blood & 1 & \\
\hline Bilirubin elevation & 3 & \\
\hline Skin itching/rashes/ puffy face & 2 & \\
\hline Nasal bleeding & 1 & 2 \\
\hline Gastric hemorrhage & 1 & 1 \\
\hline Expired & & \\
\hline Positive pregnancy test & & \\
\hline DAA: direct-acting antiviral; GLE/PIB: glecaprevir/pibrentasvir; SOF/VEL: sofosbuvir/velpatasvir \\
\hline
\end{tabular}

Table 3

Virologic responses

\begin{tabular}{|c|c|c|c|}
\hline \multirow[t]{2}{*}{ HCV RNA < LLOD } & Patient $(\mathrm{N}=742)$ & Patient $(\mathrm{N}=614)$ & \multirow[t]{2}{*}{$p$ value } \\
\hline & GLE/PIB, n /N (\%) & SOF/VEL, n /N (\%) & \\
\hline End of Treatment & & & 0.363 \\
\hline ETR (PP) & 724/727 (99.6) & $593 / 596$ (99.5) & \\
\hline Lost to follow-up & 15 & 18 & \\
\hline \multicolumn{4}{|l|}{ After Treatment } \\
\hline SVR12 (EP) & 710/742 (95.7) & $581 / 614(94.6)$ & 0.228 \\
\hline SVR12 (PP) & 710/718 (98.9) & $581 / 584(99.5)$ & 0.228 \\
\hline \multicolumn{4}{|c|}{ Reason for non-SVR ${ }_{12}, \mathrm{n}$} \\
\hline Relapse & 7 & 0 & \\
\hline Nonresponse & 1 & 3 & \\
\hline Lost to follow-up & 24 & 30 & \\
\hline
\end{tabular}


The baseline characteristics of the 11 patients who did not attain SVR12 are summarized in the Supporting Information Table S1. The majority of these patients were treatment-naïve, and only one 77year-old man with HCV genotype 2 without advanced liver fibrosis had prior treatment experience. All seven relapsers were treated with GLE/PIB, and only one had advanced liver fibrosis with an FIB-4 score of 3.87 .

Subgroup analyses for SVR12 rates by patient characteristics are shown in Fig. 2. The SVR12 rates were excellent (98.3-100\%, PP analysis) despite age, sex, HBV coinfection, prior HCC, prior treatment experience, diabetes mellitus, baseline advanced liver fibrosis or cirrhosis, and baseline HCV RNA level.

\section{Safety outcomes}

Of the 1356 patients, 1327 (97.8\%) completed the therapeutic course. Two deaths occurred during week 12 of SOF/VEL treatment: a 76-year-old woman died due to underlying decompensated liver cirrhosis with progression and a 73-year-old man due to spontaneous bacterial peritonitis with septic shock. Six deaths occurred in the off-DAA treatment group during the 12-week follow-up. The causes of death were H1N1 influenza pneumonia, septic shock with metabolic acidosis, biliary tract infection with septic shock, lung cancer with respiratory failure, out-of-hospital cardiac arrest of unknown cause, and severe sepsis; one patient died per cause. None of the deaths were attributable to the DAA treatment. The most common AEs, comprising $>3 \%$ of the total patients in the GLE/PIB and SOF/VEL groups, were as follows: pruritus ( $17.4 \%$ vs. $2.9 \%)$, abdominal discomfort ( $5.8 \%$ vs. $4.4 \%$ ), dizziness ( $4.2 \%$ vs. $2 \%$ ), fatigue ( $3.1 \%$ vs. $2.9 \%)$, and elevation of total bilirubin in $1.5-3 \times$ ULN (5.3\% vs. $2.9 \%$ ) (Table 4). Only five drug discontinuations resulted from AEs (bilirubin elevation: 3 , dermatological: 2, in the GLE/PIB group). 
Table 4

Safety Summary

\begin{tabular}{|c|c|c|c|}
\hline Event, $n(\%)$ & GLE/PIB $(\mathrm{N}=742)$ & SOF/VEL $(\mathrm{N}=614)$ & $\begin{array}{l}\text { Total } \\
(\mathrm{N}=1356)\end{array}$ \\
\hline \multicolumn{4}{|l|}{ Adverse events (> $3 \%)$} \\
\hline skin itching & $129(17.4)$ & $18(2.9)$ & $147(10.8)$ \\
\hline abdominal discomfort & $43(5.8)$ & $27(4.4)$ & $70(5.2)$ \\
\hline dizziness & $31(4.2)$ & $12(2)$ & $43(3.2)$ \\
\hline malaise & $23(3.1)$ & $18(2.9)$ & $41(3)$ \\
\hline \multicolumn{4}{|c|}{ Laboratory Adverse Event, n (\%) } \\
\hline \multicolumn{4}{|l|}{ Total bilirubin elevation } \\
\hline $1.5-3 \times$ ULN & $39(5.3)$ & $18(2.9)$ & $57(4.2)$ \\
\hline$>3 \times$ ULN & $4(0.5)$ & $6(1)$ & $10(0.7)$ \\
\hline \multicolumn{4}{|l|}{ AST elevation } \\
\hline $3-5 \times U L N$ & $3(0.4)$ & $9(1.5)$ & $12(0.9)$ \\
\hline$>5 \times$ ULN & $3(0.4)$ & $1(0.2)$ & $4(0.3)$ \\
\hline \multicolumn{4}{|l|}{ ALT elevation } \\
\hline $3-5 \times$ ULN & $6(0.8)$ & $4(0.7)$ & $10(0.7)$ \\
\hline$>5 \times U L N$ & $7(0.9)$ & $2(0.3)$ & $9(0.7)$ \\
\hline
\end{tabular}

\section{Discussion}

With the rapid development and evolution of anti-HCV drugs, pangenotypic DAAs have gradually replaced genotype-specific DAAs as first-line therapy[9]. Pangenotypic DAAs may prevent physician errors associated with unfamiliarity with genotype-specific DAAs and broaden the number of eligible patients by reducing the need for genotyping. Our results confirmed that both GLE/PIB and SOF/VEL can serve as appropriate pangenotypic DAA regimens for the treatment of HCV in general practice. In this real-world setting involving a south Taiwanese cohort, our study revealed that the overall SVR12 rates in the GLE/PIB and SOF/VEL groups were $95.7 \%$ and $94.6 \%$, respectively by EP analysis and $98.9 \%$ and $99.5 \%$, respectively by PP analysis. This therapeutic efficacy was similar to those reported in previous clinical trials, meta-analyses, and real-world reports for GLE/PIB[10-12, 19-21] and SOF/VEL[14-17, 22-24]. The real-world data currently available mainly involve the effects of a single drug. Large-scale, real-world, 
general population cohort studies comparing these two pangenotypic DAAs are limited. The government of Taiwan was the first in Asia to vigorously promote the treatment of HCV and to reimburse these two pangenotypic DAA regimens without restriction. To our knowledge, this is the largest Asian cohort study evaluating the real-world efficacy of pangenotypic DAAs for HCV treatment.

In the subgroup analyses, we evaluated the potential factors associated with SVR12 rates. Both the GLE/PIB and SOF/VEL groups exhibited excellent SVR12 rates regardless of baseline patient characteristics. This result was comparable to previous pooled analyses and other real-world reports[1924].

Regarding tolerability and safety, $726 / 742$ (97.84\%) and $601 / 614$ (97.88\%) of patients in the GLE/PIB and SOF/VEL groups, respectively, completed the treatment course. Five patients in the GLE/PIB group did not complete the treatment due to reasons which may be related to the therapy: three due to bilirubin elevation and two due to pruritus. Two deaths, which were not related to the treatment, occurred in the SOF/VEL group during the therapeutic course. The most common AE in the GLE/PIB group was pruritus, and the proportion of patients with pruritus in our study (17.4\%) was higher than that in other real-world studies $(4.7-7.8 \%)[11,19,20]$. This may be related to more chronic kidney disease patients, with higher baseline creatinine levels $(1.38 \pm 1.92 \mathrm{mg} / \mathrm{dL})$, in the GLE/PIB group. In subjects with normal renal function, GLE/PIB is mostly eliminated through the fecal-biliary route and less than $1 \%$ of the drugs are recovered in the urine[25, 26]. However, with decreasing renal function, increased GLE/PIB plasma exposure (up to 46-56\%) has been reported[27]. Previous studies also showed higher pruritus rates (12$30.5 \%)$ in patients with severe renal impairment[28-30]. Although the pruritus rate was higher in our study, only two patients discontinued treatment for this reason. The most common AE in our SOF/VEL group was abdominal discomfort (4.4\%), which was comparable to other studies[23]. Overall, this study confirmed that GLE/PIB and SOF/VEL are both well tolerated and safe for patients with chronic HCV infection in Taiwan.

There were some limitations to this study. First, genotypes 4 and 5 are rare in Taiwan, so the pangenotypic therapeutic effect of GLE/PIB and SOF/VEL for these two genotypes was not studied. Second, the majority of data for this retrospective study was collected from electronic medical records; therefore, there were potential underreporting biases for mild to moderate AEs. Third, the HCV genotypes of the seven relapsers in the GLE/PIB group were not re-checked; therefore, the possibility of repeated infection cannot be excluded. Fourth, since the CGMH is one of the referral centers of southern Taiwan, therapeutic data for these two pangenotypic DAAs from local hospitals or private clinics are needed for further study.

In conclusion, the pangenotypic DAAs, GLE/PIB and SOF/VEL, are well tolerated and show excellent SVR12 rates for patients infected with all genotypes of the HCV in real-world practice in Taiwan.

\section{Declarations}


Data Availability: Data are available from the electrical medical record of Chang Gung Memorial Hospital. Due to legal restrictions imposed by the government of Taiwan in relation to the "Personal Information Protection Act", data cannot be made publicly available. Requests for data can be sent as a formal proposal to the corresponding author.

Animal Research: Not applicable.

Consent to Participate: Written informed consent was obtained from all participants (patients) in this study.

Ethical approval: This study was approved by the Institutional Review Board of Chang Gung Medical Foundation on April 15, 2019 (IRB No.: 201900542B0). The study was conducted in accordance with the ethical principles laid down in the Declaration of Helsinki.

Clinical Trials Registration: Not applicable.

\section{Author Contributions}

Conception and design: KCC, TSC. Analysis and interpretation of data: KCC, TSC. Drafting the article: KCC. Critical revision of the article for important intellectual content: KCC, TSC. Final approval of the article: KCC, STT, KLW, CHS, YYH, WMC, YHC, CHC, CWY, HWX, WLT, CHH, SNL, TSC. Provision of study materials or patients: KCC, SYT, KLW, CHS, YYH, WMC, YHC, CHCn, CWY, HWX, WLT, CHH, SNL, TSC. Statistical expertise: TSC.

\section{Funding}

This work was supported by a research grant (CMRPG6J0171) from the Chang Gung Memorial Hospital.

Conflict of Interest: All authors declare no competing interests.

\section{Acknowledgements}

The authors thank colleagues at the Chiayi Chang Gung Memorial Hospitals who supported and assisted in laboratory analyses and collection of clinical information.

\section{References}

1. WHO. Global hepatitis report 2017. https://www.who.int/hepatitis/publications/global-hepatitisreport2017/en/ Accessed January 7, 2021.

2. Cheung MCM, Walker AJ, Hudson BE, et al. Outcomes after successful direct-acting antiviral therapy for patients with chronic hepatitis $C$ and decompensated cirrhosis. J Hepatol 2016;65:741-747.

3. Innes $\mathrm{H}$, McDonald S, Hayes $\mathrm{P}$, et al. Mortality in hepatitis $\mathrm{C}$ patients who achieve a sustained viral response compared to the general population. J Hepatol 2017;66:19-27. 
4. Kanwal F, Kramer J, Asch SM, Chayanupatkul M, Cao Y, El-Serag HB. Risk of hepatocellular cancer in HCV patients treated with direct-acting antiviral agents. Gastroenterology 2017;153:996-1005.e1.

5. Mahale P, Engels EA, Li R, et al. The effect of sustained virological response on the risk of extrahepatic manifestations of hepatitis C virus infection. Gut 2018;67:553-561.

6. Nahon P, Bourcier V, Layese R, et al. Eradication of hepatitis C virus infection in patients with cirrhosis reduces risk of liver and non-liver complications. Gastroenterology 2017;152:142-156.e2.

7. Wang LS, D'Souza LS, Jacobson IM. Hepatitis C-a clinical review. J Med Virol 2016;88:1844-1855.

8. Carrat F, Fontaine H, Dorival C, et al. Clinical outcomes in patients with chronic hepatitis $\mathrm{C}$ after direct-acting antiviral treatment: a prospective cohort study. Lancet 2019;393:1453-1464.

9. Pawlotsky JM, Negro F, Aghemo A, et al. EASL recommendations on treatment of hepatitis C: final update of the series ${ }^{\square}$ J Hepatol 2020;73:1170-1218.

10. Asselah T, Kowdley KV, Zadeikis N, et al. Efficacy of glecaprevir/pibrentasvir for 8 or 12 weeks in patients with hepatitis $C$ virus genotype 2, 4, 5, or 6 infection without cirrhosis. Clin Gastroenterol Hepatol 2018;16:417-426.

11. Forns X, Lee SS, Valdes J, et al. Glecaprevir plus pibrentasvir for chronic hepatitis $C$ virus genotype 1, $2,4,5$, or 6 infection in adults with compensated cirrhosis (EXPEDITION-1): a single-arm, open-label, multicentre phase 3 trial. Lancet Infect Dis 2017;17:1062-1068.

12. Wyles D, Poordad F, Wang S, et al. Glecaprevir/pibrentasvir for hepatitis C virus genotype 3 patients with cirrhosis and/or prior treatment experience: a partially randomized phase 3 clinical trial. Hepatology 2018;67:514-523.

13. Jonas MM, Squires RH, Rhee SM, et al. Pharmacokinetics, safety, and efficacy of glecaprevir/Ppibrentasvir in adolescents with chronic hepatitis $\mathrm{C}$ virus: part 1 of the DORA study. Hepatology 2020;71:456-462.

14. Curry MP, O'Leary JG, Bzowej N, et al. Sofosbuvir and velpatasvir for HCV in patients with decompensated cirrhosis. N Engl J Med 2015;373:2618-2628.

15. Esteban R, Pineda JA, Calleja JL, et al. Efficacy of sofosbuvir and velpatasvir, with and without ribavirin, in patients with hepatitis $\mathrm{C}$ virus genotype 3 infection and cirrhosis. Gastroenterology 2018;155:1120-1127.e4.

16. Feld JJ, Jacobson IM, Hezode C, et al. Sofosbuvir and velpatasvir for HCV genotype 1, 2, 4, 5, and 6 infection. N Engl J Med 2015;373:2599-2607.

17. Foster GR, Afdhal N, Roberts SK, et al. Sofosbuvir and velpatasvir for HCV genotype 2 and 3 infection. N Engl J Med 2015;373:2608-2617.

18. Kim NG, Kullar R, Khalil H, Saab S. Meeting the WHO hepatitis C virus elimination goal: review of treatment in paediatrics. J Viral Hepat 2020;27:762-769.

19. Lampertico P, Carrion JA, Curry M, et al. Real-world effectiveness and safety of glecaprevir/pibrentasvir for the treatment of patients with chronic HCV infection: a meta-analysis. J Hepatol 2020;72:1112-1121. 
20. Liu CH, Liu CJ, Hung CC, et al. Glecaprevir/pibrentasvir for patients with chronic hepatitis $\mathrm{C}$ virus infection: real-world effectiveness and safety in Taiwan. Liver Int 2020;40:758-768.

21. Wei L, Wang G, Alami NN, et al. Glecaprevir-pibrentasvir to treat chronic hepatitis $C$ virus infection in Asia: two multicentre, phase 3 studies-a randomised, double-blind study (VOYAGE-1) and an openlabel, single-arm study (VOYAGE-2). Lancet Gastroenterol Hepatol 2020;5:839-849.

22. Mangia A, Milligan S, Khalili M, et al. Global real-world evidence of sofosbuvir/velpatasvir as simple, effective HCV treatment: analysis of 5552 patients from 12 cohorts. Liver Int 2020;40:1841-1852.

23. Wei L, Lim SG, Xie Q, et al. Sofosbuvir-velpatasvir for treatment of chronic hepatitis $C$ virus infection in Asia: a single-arm, open-label, phase 3 trial. Lancet Gastroenterol Hepatol 2019;4:127-134.

24. Wilton J, Wong S, Yu A, et al. Real-world effectiveness of sofosbuvir/velpatasvir for treatment of chronic hepatitis $\mathrm{C}$ in British Columbia, Canada: a population-based cohort study. Open Forum Infect Dis 2020;7:ofaa055.

25. Lin CW, Dutta S, Asatryan A, et al. Pharmacokinetics, safety, and tolerability of single and multiple doses of ABT-493: a first-in-human study. J Pharm Sci 2017;106:645-651.

26. Lin CW, Dutta S, Asatryan A, et al. Pharmacokinetics, safety, and tolerability following single and multiple doses of pibrentasvir in a first-in-human study. Clin Pharmacol Drug Dev 2018;7:44-52.

27. Kosloski MP, Zhao W, Marbury TC, et al. Effects of renal impairment and hemodialysis on the pharmacokinetics and safety of the glecaprevir and pibrentasvir combination in hepatitis $\mathrm{C}$ virusnegative subjects. Antimicrob Agents Chemother 2018;62:e01990-17.

28. Atsukawa M, Tsubota A, Toyoda $\mathrm{H}$, et al. The efficacy and safety of glecaprevir plus pibrentasvir in 141 patients with severe renal impairment: a prospective, multicenter study. Aliment Pharmacol Ther 2019;49:1230-1241.

29. Hsu SJ, Chiu MC, Fang YJ, et al. Real-world effectiveness and safety of glecaprevir/pibrentasvir in Asian patients with chronic hepatitis C. J Formos Med Assoc 2019;118:1187-1192.

30. Liu CH, Yang SS, Peng CY, et al. Glecaprevir/pibrentasvir for patients with chronic hepatitis C virus infection and severe renal impairment. J Viral Hepat 2020;27:568-575.

\section{Figures}




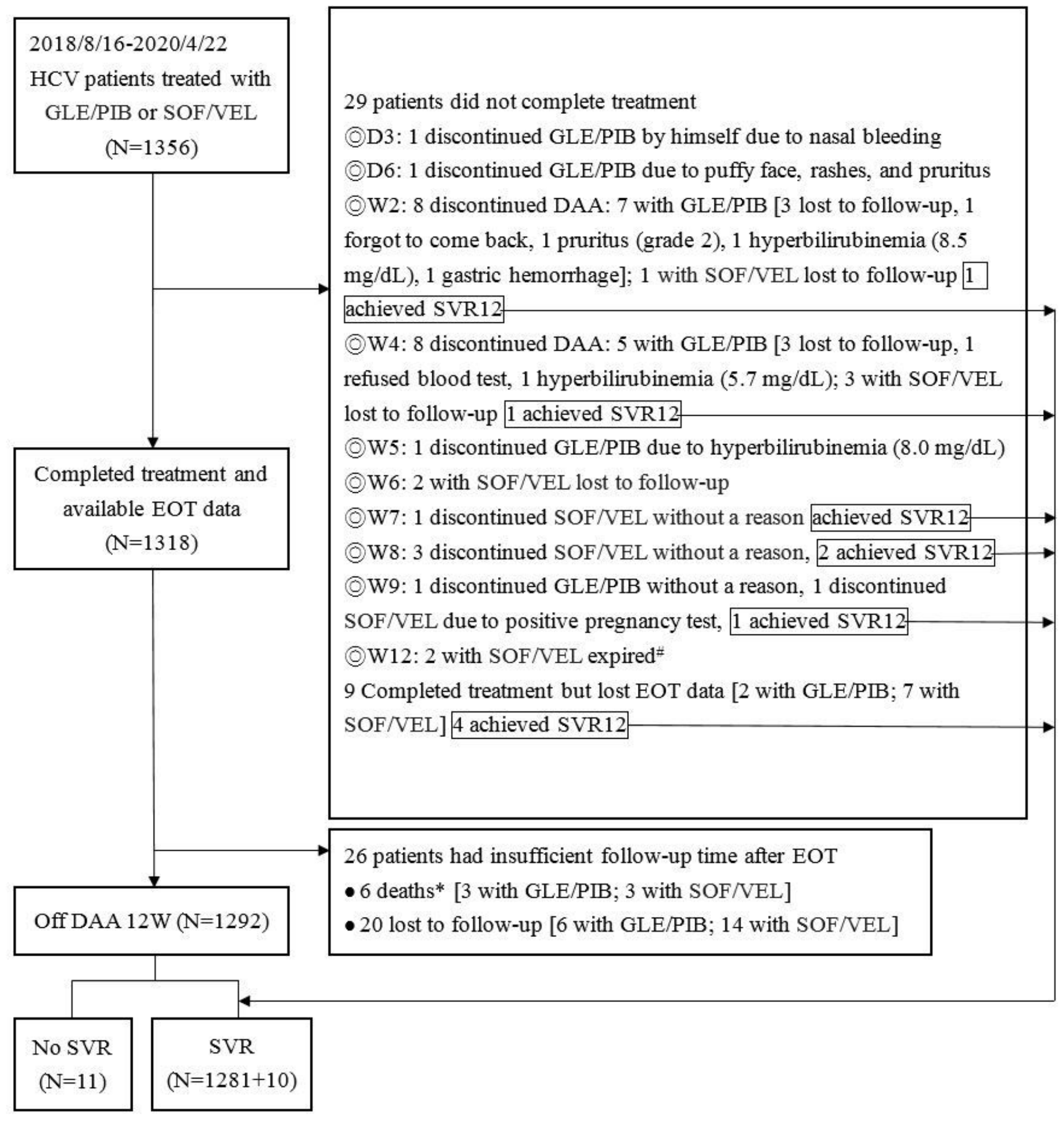

\section{Figure 1}

Study profile. \# Both died at week 12 due to underlying decompensated liver cirrhosis and related complications. * One due to H1N1 influenza pneumonia 8 weeks off DAA, Two due to sepsis 1 week and 5 weeks, respectively, off DAA, one due to biliary tract infection with septic shock 10 weeks off DAA, one due to lung cancer or pneumonia 9 weeks off DAA, and one due to out-of-hospital cardiac arrest of unknown cause 6 weeks off DAA. DAA: direct-acting antiviral; SVR12: sustained virological response 12 
weeks after treatment cessation; GLE/PIB: glecaprevir/pibrentasvir; SOF/VEL: sofosbuvir/velpatasvir; HCV: hepatitis C virus; EOT: end of treatment
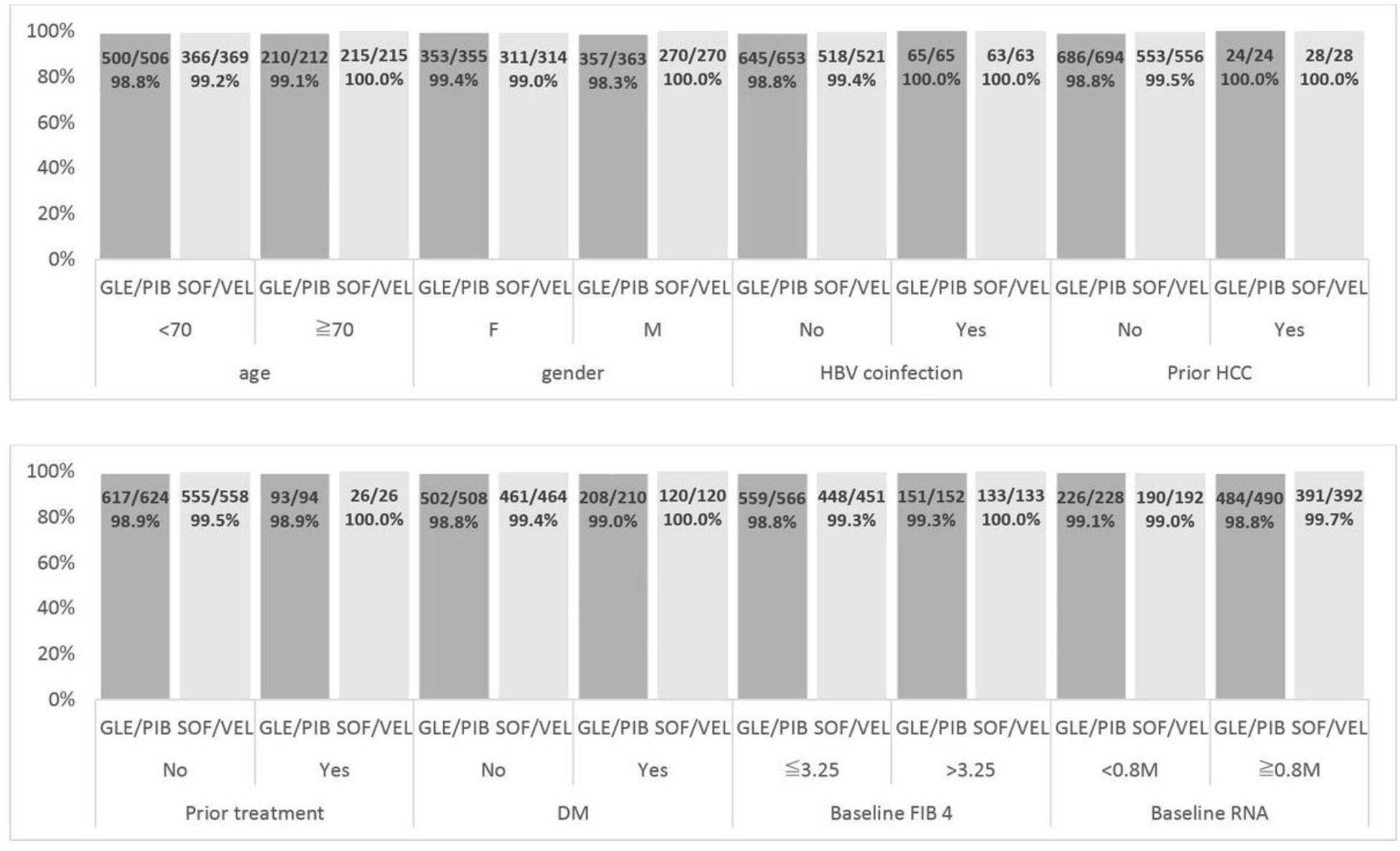

\section{Figure 2}

Subgroup analyses of SVR12 (per-protocol set). FIB-4: fibrosis index based on four factors; GLE/PIB: glecaprevir/pibrentasvir; SOF/VEL: sofosbuvir/velpatasvir; HBV: hepatitis B virus; HCC: hepatocellular carcinoma; RNA: ribonucleic acid; SVR12: sustained virological response 12 weeks after treatment cessation

\section{Supplementary Files}

This is a list of supplementary files associated with this preprint. Click to download.

- renamedeacda.docx 